\title{
Hyperbilirubinemia Diminishes Respiratory Drive in a Rat Pup Model
}

\author{
ODED MESNER, MARTHA J. MILLER, SABINE C. IBEN, PRABHA KC, CATHERINE A. MAYER, MUSA A. HAXHIU, \\ AND RICHARD J. MARTIN
}

\begin{abstract}
Department of Pediatrics [M.J.M., P.K., C.A.M., M.A.H., R.J.M.], Case Western Reserve University, Cleveland, Ohio, 44106; Division of Neonatology [O.M.], Ben-Gurion University, Beer-Sheva, Israel, 84101; Department of Pediatrics [S.C.I.], Cleveland Clinic Foundation,
\end{abstract} Cleveland, Ohio, 44195

\begin{abstract}
Although apnea is common in premature babies, there is a paucity of information concerning the pathophysiologic basis of these episodes and their relationship to other perinatal conditions such as hyperbilirubinemia. Unconjugated hyperbilirubinemia in premature infants, even in moderately high levels, may cause encephalopathy affecting brainstem functions and has been linked to increased incidence of apnea in these infants. Thus, there is a need to clarify mechanisms by which bilirubin may alter respiratory control and induce apnea of prematurity. In this study, bilirubin or placebo was infused i.v. in 9-d-old rat pups $(n=36)$. Serum hyperbilirubinemia peaked in the first hours after bilirubin infusion. Twenty-four hours after bilirubin infusion, respiration was recorded by plethysmography at rest and under hypercapnic and hypoxic conditions. In treated pups, minute ventilation in room air was significantly reduced, hyperventilatory response to $\mathrm{CO}_{2}$ was blunted, and hypoxic ventilatory depression was increased, compared with placeboinjected rat pups. Brainstem bilirubin deposition and immunoreactivity to bilirubin was detected in the brainstem on histologic analysis. We speculate that high serum bilirubin levels may cause prolonged inhibition of brainstem autonomic function and that this could underlie the exacerbation of apnea noted in premature babies who have experienced jaundice. (Pediatr Res 64: 270-274, 2008)
\end{abstract}

$\mathrm{A}$ pnea is a frequent occurrence in premature babies, affecting up to $84 \%$ of infants weighing $<1000 \mathrm{~g}$ at birth $(1,2)$. The long-term implications of this phenomenon for human development are unclear; however, some studies have suggested an association between apnea of prematurity and abnormal motor and mental development (3-5). Apneic episodes are considered to be the consequence of immature respiratory control by neuronal networks within the brainstem. However, despite advances in the field of developmental respiratory neurobiology in recent years, the pathophysiologic basis of these episodes has not yet been completely elucidated and there is a paucity of information concerning their relationship to other widespread perinatal conditions such as hyperbilirubinemia.

Hyperbilirubinemia of the newborn infant is very common, and the line separating physiologic and pathologic jaundice has

Received January 23, 2007; accepted April 18, 2008.

Correspondence: Martha J. Miller, M.D., Ph.D., Division of Neonatology, Department of Pediatrics, Rainbow Babies and Childrens Hospital, 11100 Euclid, Cleveland, Ohio 44106; e-mail:mjm8@case.edu

Supported by an "Advancing Newborn Medicine" Grant from INO Therapeutics, HL 62527 and HL50527. been difficult to define (6). In severe cases this condition can be harmful due to the risk of bilirubin encephalopathy or development of kernicterus (7). In premature infants, in particular, bilirubin encephalopathy and kernicterus have been shown to occur at a lower threshold of serum bilirubin, compared with term babies $(8,9)$. Furthermore, an association between moderate hyperbilirubinemia and poorer neurodevelopmental outcomes has been suggested in a large, retrospective multicenter study of premature infants (10). A preliminary observation from our laboratory by DiFiore et al. suggests that a significant relationship exists between apnea of prematurity and the prior occurrence of hyperbilirubinemia (DiFiore JM et al., Persistent apnea of prematurity among very low birth weight infants: demographic and neonatal correlates. 2004, PAS Annual Meeting, May 1-4, 2004, San Francisco, California, Abstract 2991). Amin et al. (11) have also reported a correlation between bilirubin encephalopathy in premature infants and the subsequent occurrence of apneic events. Based on these findings, we designed this study to clarify the possible sustained effect of transient hyperbilirubinemia on respiratory control and apnea of prematurity in a rat pup model. We hypothesized that ventilatory control, including the responses to hypercapnia and hypoxia, would be altered in exposed pups $24 \mathrm{~h}$ after the administration of bilirubin. Such an effect could have implications for management of hyperbilirubinemia in premature babies.

\section{METHODS}

All protocols for research met with the previous approval of the institutional animal care and use committee of Case Western Reserve University in accordance with the Public Health Services Policy on humane care and use of animals.

Materials. Bilirubin and BSA were purchased from Sigma Chemical (St. Louis, MO). Gas mixtures of $5 \% \mathrm{O}_{2}$ balance $\mathrm{N}_{2}$ and $10 \% \mathrm{CO}_{2}, 30 \% \mathrm{O}_{2}$, balance $\mathrm{N}_{2}$ were obtained from a commercial supplier and were of medical grade. Monoclonal anti-bilirubin antibodies (24G7) were purchased from Shino-Test Company, Tokyo, Japan. Goat anti-mouse IgG was purchased from Jackson ImmunoResearch Laboratories (West Grove, PA). Sprague-Dawley rat pup litters were obtained from Harlan Laboratories, Indianapolis, IN.

Preparation of bilirubin and placebo solutions. Bilirubin was dissolved in $0.1 \mathrm{M} \mathrm{NaOH}$, stabilized with BSA (molar ratio bilirubin:albumin 14:1), and

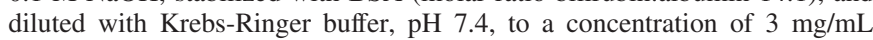
bilirubin and $24 \mathrm{mg} / \mathrm{mL}$ albumin (final $\mathrm{pH}$ 7.7-7.9), as described by Hansen et al. (12). The placebo solution was comprised of BSA, $24 \mathrm{mg} / \mathrm{mL}$ in Krebs-Ringer buffer, $\mathrm{pH} 7.5$.

The rat model. At $9 \mathrm{~d}$ of age, the pups were weighed, and then anesthetized with an intraperitoneal injection of $3-5 \mathrm{~mL} / \mathrm{kg}$ of a mixture of ketamine and xylazine ( 5 and $1 \mathrm{mg} / \mathrm{mL}$, respectively). Anesthesia was maintained throughout the preparation procedure by supplementary injections of $1-2 \mathrm{~mL} / \mathrm{kg}$ as 
needed. The internal jugular vein was exposed and a silastic catheter (ID $=$ $0.3 \mathrm{~mm}, \mathrm{OD}=0.64 \mathrm{~mm}$ ) was inserted and sutured in place. The pups were then injected i.v. with either bilirubin $50 \mathrm{mg} / \mathrm{kg}$ (in solution, $17 \mu \mathrm{L} / \mathrm{g}$ rat weight) or the same volume of the placebo solution, over $5 \mathrm{~min}$. The dose of bilirubin used was based on the studies of Hansen et al. (12). All bilirubin containing tubes, syringes, and lines were protected from light.

The pups were subsequently divided into two groups. In one group $(n=$ 18), pups were killed at 10-180 min after the end of the bilirubin infusion for determination of serum bilirubin levels. Pups in the other group $(n=6$ treated and 6 placebo) were returned to their mother and nursed until the next day, when respiratory measurements were performed $24 \mathrm{~h}$ after bilirubin injection. At this time, the pups were mobile, and were able to suckle from the mother. An additional six treated pups were killed with i.v. ketamine and xylazine, 20 $\mathrm{mL} / \mathrm{kg}$, for histologic analysis of the brain. The pups were perfused transcardially with a calcium-free tyrode solution and then with $4 \%$ paraformaldehyde in PBS, pH 7.4. The brain was removed, postfixed in $4 \%$ paraformaldehyde, and cryoprotected for $24 \mathrm{~h}$ in $30 \%$ sucrose in PBS before further processing. A subset of six brains was rapidly frozen on dry ice, and sectioned on a cryostat (Leica 2000S) to optimize visualization of yellow bilirubin pigment within the tissue.

Analytical methods. Serum bilirubin and albumin were analyzed with the Dimension RXL clinical chemistry system (Dade Behring, Springfield, IL). This system uses the Diazo method to determine total serum bilirubin and the bromocresol purple dye-binding method to determine serum albumin levels.

Measurement of respiration. Twenty-four hours after the i.v. injection of bilirubin or placebo, pups were placed in a heated, flow-through barometric plethysmograph (Buxco Electronics, Troy, NY). We chose to perform the plethysmography at this time after injection of bilirubin to avoid possible transient effects of anesthesia on respiration. Bilirubin-injected pups $(n=6)$ were compared with placebo-injected pups $(n=6)$. The pups were allowed to adapt to the plethysmograph until they were quiet with little or no spontaneous movement. Then, after baseline respiration was measured for $10 \mathrm{~min}$, the respiratory responses to hypercapnia and hypoxia were tested. To evaluate the response of breathing to an increase in inhaled $\mathrm{CO}_{2}$ above this level (hypercapnia), the inflow to the plethysmograph was switched to the test gas: $10 \%$ $\mathrm{CO}_{2}, 30 \% \mathrm{O}_{2}$, balance $\mathrm{N}_{2}$ (to prevent bronchoconstriction) (13) for $10 \mathrm{~min}$, after which the pups were allowed to return to their baseline minute ventilation in room air. To evaluate the response of the rat pup to severe hypoxia, the pup was exposed to $5 \%$ inspired oxygen, balance $\mathrm{N}_{2}$ for $5 \mathrm{~min}$. At the conclusion of the test, the plethysmograph gas flow was returned to room air. Minute ventilation (the product of respiratory rate and tidal volume) was calculated. Minute ventilation was averaged over 20-s intervals before and during the test gas exposure, and expressed as $\mathrm{mL} / \mathrm{min} / \mathrm{g}$ weight of the rat.

Immunohistochemistry. Brain sections were washed for $1 \mathrm{~h}$ in $\mathrm{PBS}(\mathrm{pH} 7.4)$ to remove traces of fixative and then the tissues were placed in $0.5 \%$ hydrogen peroxide for $30 \mathrm{~min}$ to quench endogenous peroxidase. The tissues were washed in PBS 3 times, $10 \mathrm{~min}$ each, and then immersed for $1 \mathrm{~h}$ in a solution of 5\% normal goat serum to block nonspecific binding of antibodies. The tissues were then incubated overnight in mouse MAb to bilirubin (diluted 1:125 in PBS). The antibody to bilirubin recognizes the conjugated and nonconjugated forms of bilirubin IXa but not other isobilirubins (14). The sections were then washed in three changes of PBS and incubated for $2 \mathrm{~h}$ at room temperature in a 1:200 dilution of biotinylated goat anti-mouse IgG. This was followed by three changes of PBS to remove unreacted secondary antibody. The sections were then reacted for $1 \mathrm{~h}$ at room temperature in avidin-biotin complex. The reaction was visualized by treatment for $2 \mathrm{~min}$ in $0.05 \%$ 3,3-diaminobenzidine tetrahydrochloride solution in tris-buffered saline (TBS; pH 7.6) containing $0.05 \%$ hydrogen peroxide. The color reaction was stopped with several washes of TBS. Some sections were mounted on gelatin-coated glass slides, and lightly counterstained with neutral red, dehydrated in alcohol series, then cleared in xylene before coverslipping. Control sections were incubated in PBS containing normal goat serum instead of primary antibody.

Statistical analysis. Baseline mean minute ventilation in the bilirubintreated versus the placebo group was analyzed with a two-tailed unpaired $t$ test. The responses to hypercapnia and hypoxia in the two groups were analyzed using two-way ANOVA with correction for repeated measures. The level of significance was $p \leq 0.05$. Results are expressed as mean \pm SEM.

\section{RESULTS}

Serum bilirubin and albumin levels. The infusion of bilirubin transiently increased bilirubin levels to as high as 25 $\mathrm{mg} / \mathrm{dL}$, tapering to $<10 \mathrm{mg} / \mathrm{dL}$ at $60 \mathrm{~min}$ postinfusion, $<5$ $\mathrm{mg} / \mathrm{dL}$ at $180 \mathrm{~min}$, and $<1 \mathrm{mg} / \mathrm{dL}$ at $24 \mathrm{~h}$ (Fig. 1). Albumin levels of all pups were at $<1 \mathrm{~g} / \mathrm{dL}(0.2-0.8)$. The peak serum

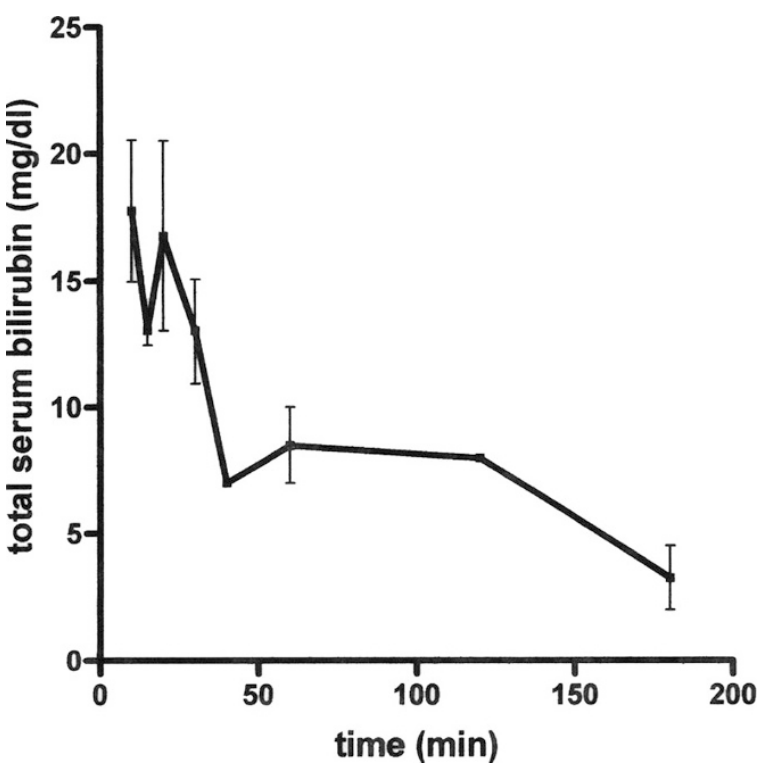

Figure 1. Total serum bilirubin in rat pups. Serum bilirubin level was maximum at 10 min after injection (mean \pm SEM).

levels observed were within the range found in infants with jaundice (15).

Minute ventilation. Twenty-four hours after the injection of bilirubin or placebo, the pups' baseline minute ventilation was measured in room air, after they had adapted to the plethysmograph. The bilirubin-injected group had significantly reduced minute ventilation at rest, when compared with the placeboinjected group, $3.20 \pm 0.51$ versus $3.56 \pm 0.52 \mathrm{~mL} / \mathrm{min} / \mathrm{g}$, respectively, $p \leq 0.02$. Subsequently, the inflow to the plethysmograph was switched to the test gas: $10 \% \mathrm{CO}_{2}, 30 \% \mathrm{O}_{2}$, balance $\mathrm{N}_{2}$ for $10 \mathrm{~min}$. As expected, all pups responded to the resultant hypercapnia by increasing their minute ventilation but this hyperventilatory response was significantly blunted in the bilirubin-injected pups ( $p<0.001$ ) (Fig. 2). When the pups were exposed to severe hypoxic conditions $\left(5 \% \mathrm{O}_{2}\right)$, we observed an increase in minute ventilation in the placebo-infused pups, which lasted $10 \mathrm{~min}$. In the bilirubin-infused pups there was no statistically significant initial increase in ventilation during the first 2

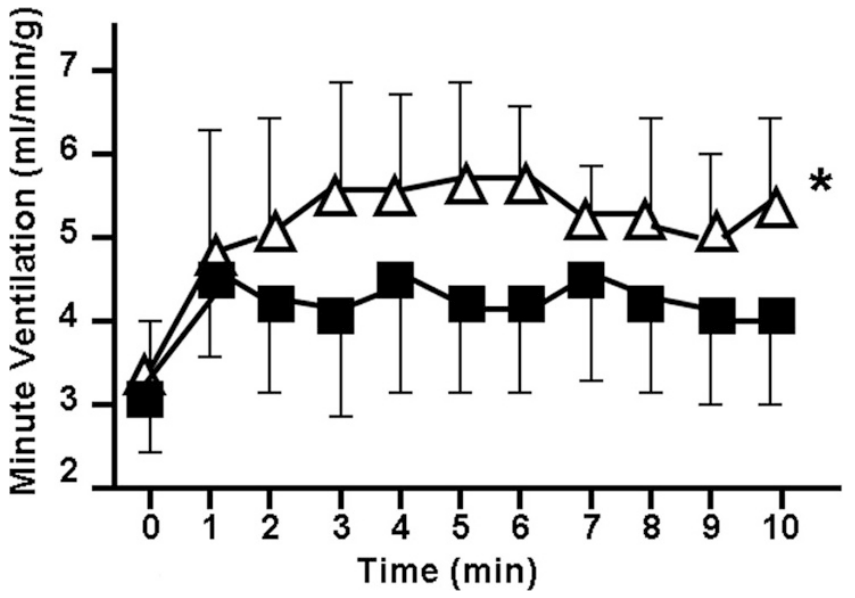

Figure 2. Hyperbilirubinemia decreased the ventilatory response to hypercapnia in 9-d-old rat pups. Hypercapnia began at T0. $(\triangle)$ Placebo-treated group, $(\square)$ bilirubin-treated group, $* p<0.001$, mean \pm SEM. 
min of exposure, and thereafter ventilation decreased to below the baseline, a response which differed significantly from the placebo, treated pups ( $p<0.001$ ) (Fig. 3).

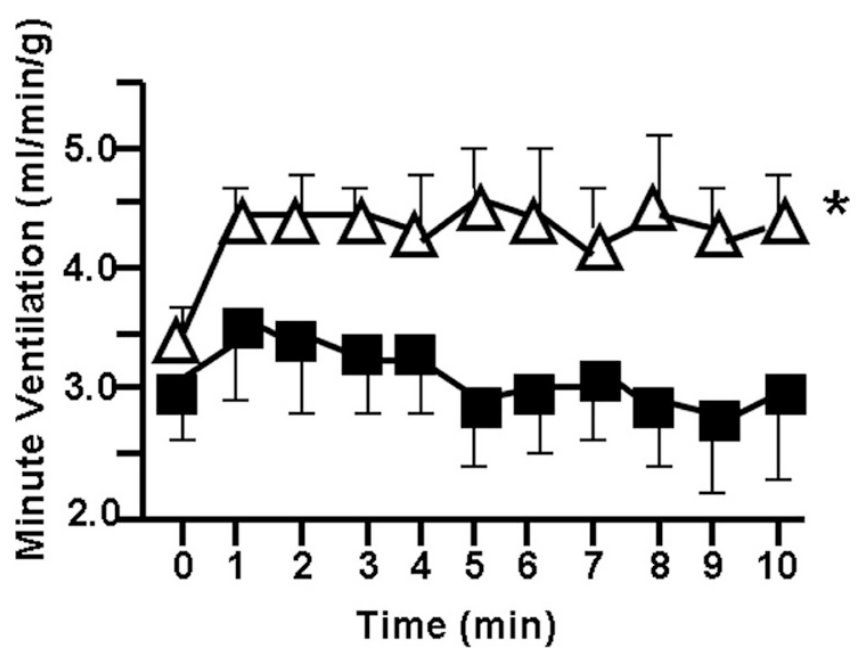

Figure 3. Hyperbilirubinemia decreased the ventilatory response to hypoxia in 9-d-old rat pups. Hypoxia began at T0. $(\triangle)$ Placebo-treated group, ( bilirubin-treated group, ${ }^{*} p<0.001$, mean \pm SEM.

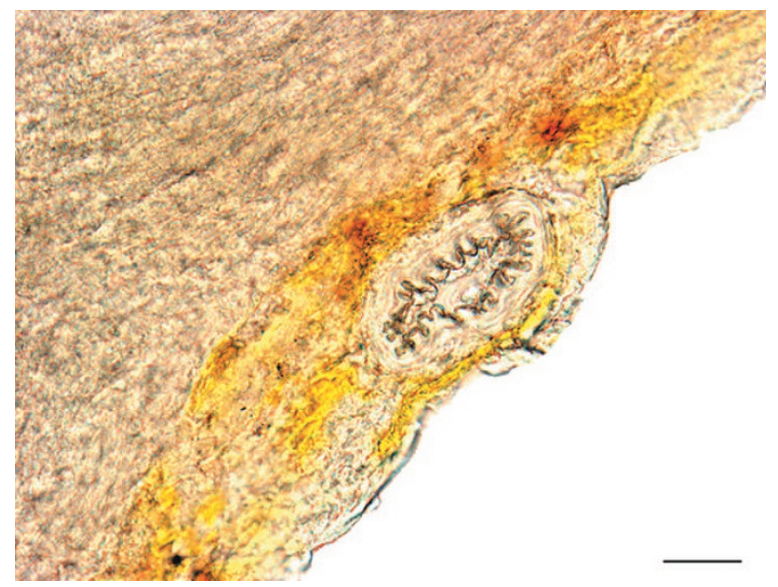

Figure 4. On histologic examination, accumulation of bilirubin pigment was observed in tissue from the ventral surface of the caudal medulla adjacent to the basillary artery. Scale bar $=20 \mu \mathrm{m}$.
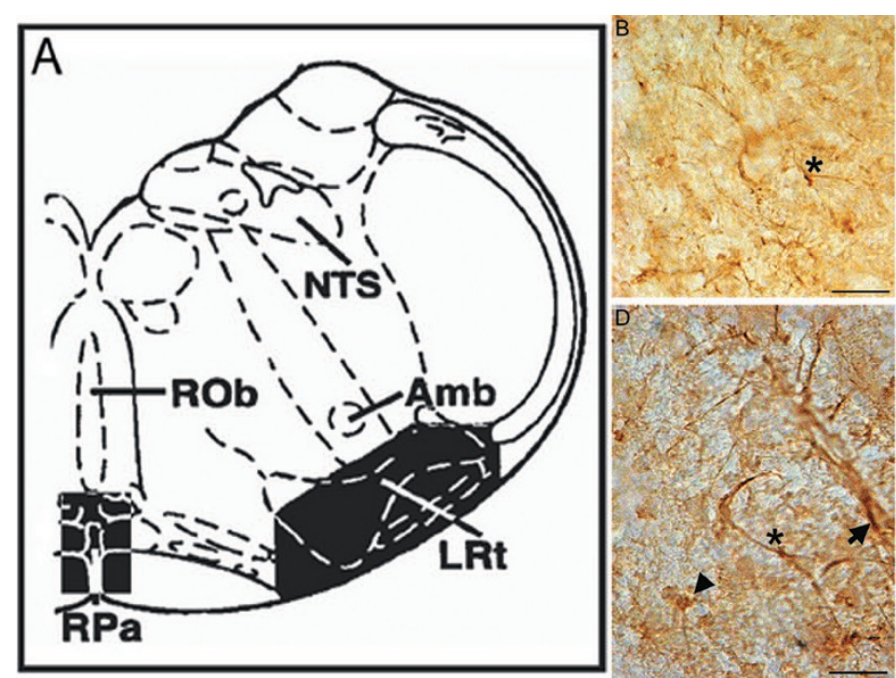

Histology. Histologic analysis focused on the medulla oblongata within which lie neuronal networks involved in cardiorespiratory control. In this study, yellow bilirubin pigment and crystals were directly visualized in the brains of pups, killed 10-30 min after the injection of bilirubin, in nonfixed sections. Bilirubin accumulation was demonstrated in the ventral surface of the medulla, adjacent to the basillary artery, in parapyramidal regions and in the raphe pallidus nucleus area (Figs. 4 and 5E).

Immunohistochemistry. We immunostained with antibody to bilirubin to examine its localization within the ventral surface of the medulla in treated pups. The most intense binding of antibody to bilirubin was demonstrated during the acute hyperbilirubinemic stage, 10-30 min after the injection of bilirubin. Immunostaining for bilirubin was found in neurons, astrocytes, and nerve fibers on the surface and within the parenchyma of that region (Fig. $5 B-D$ ).

\section{DISCUSSION}

Preterm infants exhibit immaturity of respiratory control, exemplified by a high incidence of apnea and periodic breathing. These disorders of autonomic control of breathing appear to be due to inhibitory influences of central origin in the premature neonate, which persist from the fetal state. Among the manifestations of this influence are the lower response to $\mathrm{CO}_{2}$ and paradoxical biphasic response to hypoxia of the premature infant when compared with the older child. The diminished ventilatory response to $\mathrm{CO}_{2}$ of preterm infants, especially those with apnea, could be a primary mechanism contributing to apnea of prematurity (16). Although apnea is a function of immature development of autonomic control of breathing, it is unclear if pathophysiologic conditions of prematurity aggravate this disorder by disturbing normal brainstem autonomic function.

Neonatal jaundice is a common but potentially harmful condition due to the neurotoxic effect of bilirubin, which may cause bilirubin encephalopathy or kernicterus in severe cases. Bilirubin encephalopathy and kernicterus are associated with brainstem dysfunction including sensorineural hearing loss and apnea (11). As evidence of this dysfunction, brainstem-

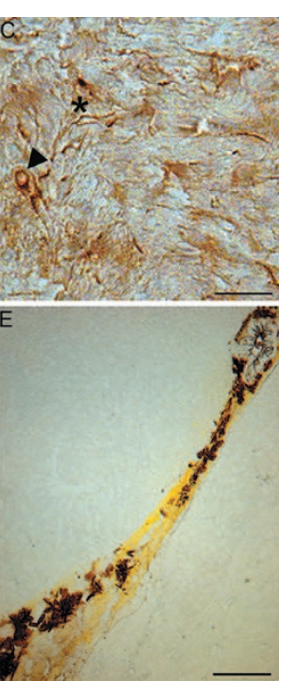

Figure 5. Examples of bilirubin detected by light microscopy and immunohistochemistry in the rat pup caudal medulla. (A) Schematic diagram of the medulla. Highlighted areas are imaged in panels $B-D$. In panels $B-D$, immunostaining for bilirubin is visualized with diaminobenzidine (brown). Arrowheads: immunostained neurons; arrows: vessels; asterisks: cell processes. (B) Raphe pallidus. (C) Raphe obscurus. (D) Ventrolateral medullary surface. (E) Ventrolateral medullary surface showing intravascular bilirubin crystals and pigment, $15 \mathrm{~min}$ postinfusion (unfixed, nonstained tissue). Scale bar: $B-D=20 \mu \mathrm{m}$, and $E=10 \mu \mathrm{m}$. $\mathrm{RPa}$, raphe pallidus nucleus; LRt, lateral reticular nucleus; Rob, raphe obscurus nucleus; NTS, nucleus tractus solitarius; Amb, ambiguus nucleus. 
evoked response has been shown to be abnormal both in jaundiced rats (17) and in premature infants with hyperbilirubinemia $(8,18)$, in whom it has been proposed as a useful tool for the clinical assessment of bilirubin-induced neurotoxicity. Furthermore, in a study on premature infants with hyperbilirubinemia, Amin et al. (11) found more concurrent apneic events and higher requirement for respiratory support in the infants with transient bilirubin encephalopathy, as defined by abnormal auditory brainstem-evoked response. The relationship between hyperbilirubinemia and apnea in their study was most pronounced during the first week of life. These findings suggest a potential link between hyperbilirubinemia, abnormal brainstem function, and apnea in premature infants.

To characterize the relationship between demographic and neonatal risk factors and apnea of prematurity, DiFiore et al. performed a multivariate logistic regression analysis based on 12-h cardiorespiratory monitoring and inductance plethysmography. This study showed a significant relationship between apnea and Caucasian race, postconceptual age, and the occurrence of peak bilirubin $(>10 \mathrm{mg} / \mathrm{dL})$, at a mean age of $8.4 \mathrm{wk}$, long after hyperbilirubinemia had resolved. Based on these findings, we hypothesized that hyperbilirubinemia could cause a sustained alteration of respiratory control. Therefore, we developed the animal model used in this study to investigate the possible correlation between hyperbilirubinemia and abnormalities of respiratory control in the immature nervous system. We chose young rat pups at d 9 in whom autonomic control of breathing is still developing, as in the human premature infant (19). Hansen et al. (12) have previously demonstrated rapid elevation of serum bilirubin levels and acute entry of bilirubin into rat brain cells following an i.v. infusion of $50 \mathrm{mg} / \mathrm{kg}$ of a bilirubin solution. We injected a similar preparation of bilirubin and documented a similar surge in serum bilirubin levels, followed by a rapid drop and resolution of the hyperbilirubinemia within hours after the injection.

The rat pups in the bilirubin-injected group had diminished minute ventilation $24 \mathrm{~h}$ after bilirubin exposure, compared with their placebo-injected littermates, a diminished hyperventilatory response to hypercapnia and greater ventilatory depression in response to hypoxia. These effects represent one aspect of the encephalopathic responses to bilirubin, whose severity and duration depend on the persistence of bilirubin in brain tissues and the cellular and molecular mechanisms impacted. Toxicity may range from mild to severe, depending on the maturity of the affected individual and the level of bilirubin in the brain. Hypothetically, cytopathic effects of bilirubin could also occur in the peripheral nervous system at the level of the peripheral chemoreceptors, the carotid bodies, which are critical for the normal increase in ventilation which occurs in response to hypoxia (20).

At low levels bilirubin is a potent antioxidant and may be neuroprotective (21), but at high levels toxicity occurs, including glutamate excitotoxicity due to activation of NMDA receptors, alteration of dopamine uptake and synthesis, mitochondrial damage, induction of apoptosis, and cell death (22-27). The sensitivity and reversibility of each of these toxic actions in the individual cellular constituents of the brain are active areas of ongoing investigation. We neither know which of these processes impact the brain of the human or the rat, nor the time course of recovery of each. Nor is it known whether toxic levels of bilirubin could alter the complex balance of neurotransmission involved in peripheral chemoreceptor function (28). The survival of the large majority of infants with jaundice testifies to the reversibility of many of the effects of bilirubin, but the details of cellular and functional recovery remain incompletely understood at this time.

Our study was conducted in a developing rat model, which exhibits some differences from the immature human. Specifically, spontaneous, prolonged apnea does not occur in rat pups; hence, a direct effect of bilirubin on apnea of prematurity could not be demonstrated in this study. However, abnormalities in respiratory control, including an altered hypercapnic response, have been observed in apneic infants (16). Therefore, we speculate that bilirubin-induced damage to autonomic pathways in the brainstem or peripheral nervous system could contribute to vulnerability to sleep apnea in previously jaundiced premature infants.

In this study, measurement of respiratory function occurred at $24 \mathrm{~h}$ after bilirubin injection. It remains to be shown whether lower doses of bilirubin than that we used could produce alteration of control of breathing, and whether disordered breathing would persist for a more prolonged period in the developing rat pup. Furthermore, detailed exploration of the dose-response to hypercapnia, as well as the response of the pups to intermittent hypoxia could better define the alteration in ventilatory control produced by hyperbilirubinemia.

In light of our observations, we speculate that there may be a role for the treatment of hyperbilirubinemia as a mechanism for diminishing the incidence of apnea in premature infants. This possible correlation requires further study both in animal models and in clinical trials.

Acknowledgments. We thank Shijian Liu for technical support in tissue preparation and histology. We greatly appreciate the assistance of Michael Kaplan, Jerusalem, Israel, and Thor W.R. Hansen, Oslo, Norway, in the design of this study.

\section{REFERENCES}

1. Barrington KJ, Finer N, Li D 1996 Predischarge respiratory recordings in very low birth weight newborn infants. J Pediatr 129:934-940

2. Alden ER, Mandelkorn T, Woodrum DE, Wennberg RP, Parks CR, Hodson WA 1972 Morbidity and mortality of infants weighing less than 1000 grams in an intensive care nursery. Pediatrics 50:40-49

3. Cheung PY, Barrington KJ, Finer NN, Robertson CM 1999 Early childhood neurodevelopment in very low birth weight infants with predischarge apnea. Pediatr Pulmonol 27:14-20

4. Hunt CE, Corwin MJ, Baird T, Tinsley LR, Palmer P, Ramanathan R, Crowell DH, Schafer S, Martin RJ, Hufford D, Peucker M, Weese-Mayer DE, Silvestri JM, Neuman MR, Cantey-Kiser J 2004 Cardiorespiratory events detected by home memory monitoring and one-year neurodevelopmental outcome. J Pediatr 145:465471

5. Taylor HG, Klein N, Schatschneider C, Hack M 1998 Predictors of early school age outcomes in very low birth weight children. J Dev Behav Pediatr 19:235-243

6. Maisels MJ 2006 What's in a name? Physiologic and pathologic jaundice: the conundrum of defining normal bilirubin levels in the newborn. Pediatrics 118:805807

7. Watchko JF, Claassen D 1994 Kernicterus in premature infants: current prevalence and relationship to NICHD phototherapy study exchange criteria. Pediatrics 93:996999

8. Amin SB 2004 Clinical assessment of bilirubin-induced neurotoxicity in premature infants. Semin Perinatol 28:340-347 
9. Govaert P, Lequin M, Swarte R, Robben S, De Coo R, Weisglas-Kuperus N, De Rijke Y, Sinaasappel M, Barkovich J 2003 Changes in globus pallidus with (pre)term kernicterus. Pediatrics 112:1256-1263

10. Oh W, Tyson JE, Fanaroff AA, Vohr BR, Perritt R, Stoll BJ, Ehrenkranz RA, Carlo WA, Shankaran S, Poole K, Wright LL 2003 Association between peak serum bilirubin and neurodevelopmental outcomes in extremely low birth weight infants. Pediatrics 112:773-779

11. Amin SB, Charafeddine L, Guillet R 2005 Transient bilirubin encephalopathy and apnea of prematurity in 28 to 32 weeks gestational age infants. J Perinatol 25:386390

12. Hansen T, Tommarello S, Allen J 2001 Subcellular localization of bilirubin in rat brain after in vivo i.v. administration of [3H]bilirubin. Pediatr Res 49:203-207

13. Dewachter P, Saunier CG, Duvivier C, Peslin R, Laxenaire MC 1992 Changes in inspired gas composition and experimental bronchospasm in the rabbit. Respir Physiol 90:261-269

14. Izumi Y, Yamazaki M, Shimizu S, Shimizu K, Yamaguchi T, Nakajima H 1988 Anti-bilirubin monoclonal antibody. II. Enzyme-linked immunosorbent assay for bilirubin fractions by combination of two monoclonal antibodies. Biochim Biophys Acta 967:261-266

15. Schneider AP II 1986 Breast milk jaundice in the newborn. A real entity. JAMA 255:3270-3274

16. Gerhardt T, Bancalari E 1984 Apnea of prematurity. I. Lung function and regulation of breathing. Pediatrics 74:58-62

17. Shapiro SM 2002 Somatosensory and brainstem auditory evoked potentials in the Gunn rat model of acute bilirubin neurotoxicity. Pediatr Res 52:844-849
18. Henderson-Smart DJ, Pettigrew AG, Campbell DJ 1983 Clinical apnea and brainstem neural function in preterm infants. N Engl J Med 308:353-357

19. Abu-Shaweesh JM 2004 Maturation of respiratory reflex responses in the fetus and neonate. Semin Neonatol 9:169-180

20. Prabhakar NR 2000 Oxygen sensing by the carotid body chemoreceptors. J Appl Physiol 88:2287-2295

21. Dore S, Takahashi M, Ferris CD, Zakhary R, Hester LD, Guastella D, Snyder SH 1999 Bilirubin, formed by activation of heme oxygenase-2, protects neurons against oxidative stress injury. Proc Natl Acad Sci USA 96:2445-2450

22. Ostrow JD, Pascolo L, Brites D, Tiribelli C 2004 Molecular basis of bilirubininduced neurotoxicity. Trends Mol Med 10:65-70

23. Silva RF, Rodrigues CM, Brites D 2002 Rat cultured neuronal and glial cells respond differently to toxicity of unconjugated bilirubin. Pediatr Res 51:535-541

24. Rodrigues CM, Sola S, Brites D 2002 Bilirubin induces apoptosis via the mitochondrial pathway in developing rat brain neurons. Hepatology 35:1186-1195

25. McDonald JW, Shapiro SM, Silverstein FS, Johnston MV 1998 Role of glutamate receptor-mediated excitotoxicity in bilirubin-induced brain injury in the Gunn rat model. Exp Neurol 150:21-29

26. Amato MM, Kilguss NV, Gelardi NL, Cashore WJ 1994 Dose-effect relationship of bilirubin on striatal synaptosomes in rats. Biol Neonate 66:288-293

27. Ochoa EL, Wennberg RP, An Y, Tandon T, Takashima T, Nguyen T, Chui A 1993 Interactions of bilirubin with isolated presynaptic nerve terminals: functional effects on the uptake and release of neurotransmitters. Cell Mol Neurobiol 13:69-86

28. Gauda EB, Bamford O, Gerfen CR 1996 Developmental expression of tyrosine hydroxylase, D2-dopamine receptor and substance $\mathrm{P}$ genes in the carotid body of the rat. Neuroscience 75:969-977 